\title{
Control over size, shape, and photonics of self-assembled organic nanocrystals
}

\author{
Chen Shahar ${ }^{1}$, Yaron Tidhar ${ }^{1}$, Yunmin Jung ${ }^{2,3}$, Haim Weissman ${ }^{1}$, Sidney R. Cohen ${ }^{4}$, \\ Ronit Bitton ${ }^{5,6}$, Iddo Pinkas ${ }^{4}$, Gilad Haran ${ }^{2}$ and Boris Rybtchinski ${ }^{* 1}$
}

\section{Full Research Paper}

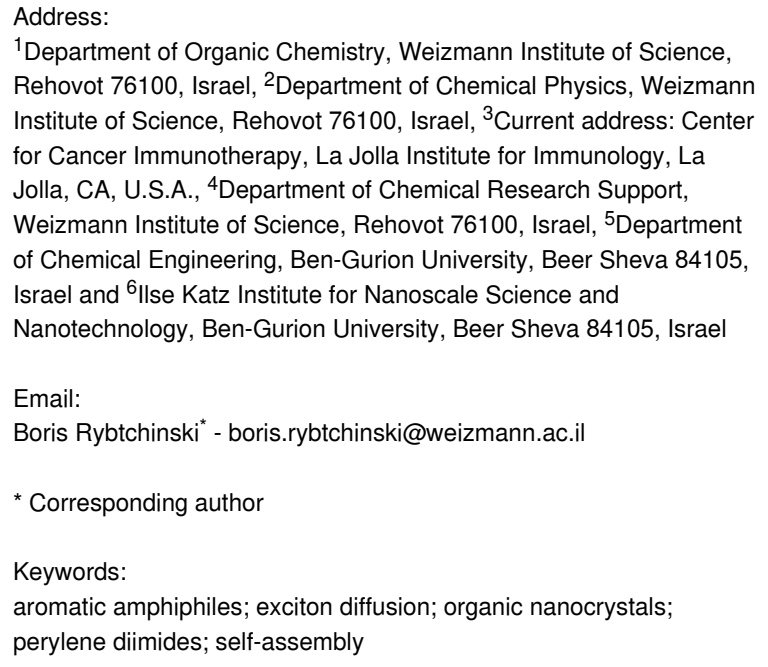

${ }^{1}$ Department of Organic Chemistry, Weizmann Institute of Science, Rehovot 76100, Israel, ${ }^{2}$ Department of Chemical Physics, Weizmann Institute of Science, Rehovot 76100 , Israel, ${ }^{3}$ Current address: Center for Cancer Immunotherapy, La Jolla Institute for Immunology, La Jolla, CA, U.S.A., ${ }^{4}$ Department of Chemical Research Support, Weizmann Institute of Science, Rehovot 76100, Israel, ${ }^{5}$ Department of Chemical Engineering, Ben-Gurion University, Beer Sheva 84105, Israel and ${ }^{6}$ Ilse Katz Institute for Nanoscale Science and Nanotechnology, Ben-Gurion University, Beer Sheva 84105, Israel

Email:

Boris Rybtchinski* - boris.rybtchinski@weizmann.ac.il

* Corresponding author

Keywords:

aromatic amphiphiles; exciton diffusion; organic nanocrystals;

perylene diimides; self-assembly

Beilstein J. Org. Chem. 2021, 17, 42-51.

https://doi.org/10.3762/bjoc. 17.5

Received: 07 September 2020

Accepted: 03 December 2020

Published: 06 January 2021

This article is part of the thematic issue "Molecular recognition" and is dedicated to the memory of Carsten Schmuck.

Guest Editor: J. Niemeyer

(C) 2021 Shahar et al.; licensee Beilstein-Institut. License and terms: see end of document.

\begin{abstract}
The facile fabrication of free-floating organic nanocrystals (ONCs) was achieved via the kinetically controlled self-assembly of simple perylene diimide building blocks in aqueous medium. The ONCs have a thin rectangular shape, with an aspect ratio that is controlled by the content of the organic cosolvent (THF). The nanocrystals were characterized in solution by cryogenic transmission electron microscopy (cryo-TEM) and small-angle X-ray scattering. The ONCs retain their structure upon drying, as was evidenced by TEM and atom force microscopy. Photophysical studies, including femtosecond transient absorption spectroscopy, revealed a distinct influence of the ONC morphology on their photonic properties (excitation energy transfer was observed only in the high-aspect ONCs). Convenient control over the structure and function of organic nanocrystals can enhance their utility in new and developed technologies.
\end{abstract}

\section{Introduction}

Semiconductor and metal nanoparticles exhibit size- and morphology-dependent properties arising from confinement effects and strong interactions between neighboring atoms [1-3]. The correlation between nanoparticle size and the related electronic and optical properties has extensively been studied, leading to applications in novel technologies and devices [4-6]. The devel- 
opment of the reprecipitation method [7] allowed the facile fabrication of (often crystalline) organic nano- and microparticles based on polydiacetylene [8], pyrazoline [9], perylene [10], and other molecules. In several cases, size-dependent absorption was reported [11-13]. These crystals found use in optoelectronic materials [14-16], as markers for imaging applications $[12,13]$, and demonstrated anticancer properties [17]. However, control over the size and shape in such systems is challenging [8-19].

Surface chemistry methodologies allow improved control over crystalline product formation; however, these methods are indirect and limited by the nature of the interface involved in the process. For example, well-defined two-dimensional nanocrystals were obtained by the vapor transport method, resulting in improved charge mobility [20], but no control over the crystal size and morphology was demonstrated. Using self-assembled monolayers as templates for the seeding and growth of molecular crystals may offer control over structure and polymorphism [21]. However, in this method, the crystal formation is limited by the monolayer surface so that it does not allow facile bulk fabrication and restricts control over the crystal morphology $[22,23]$. Crystalline nanobelts assembled from perylene and perylene diimide (PDI) derivatives were reported, but their size and shape could not be controlled [24,25]. Modification of the building blocks in such systems results in a certain degree of control [26,27], yet the PDI nanobelts do not remain freefloating in solution and normally are characterized as solid-state materials [28], limiting the processability of the nanocrystals, the control over the morphology, and insights into the assembly.

In general, gaining control over the crystal formation represents a long-standing challenge [29-32]. In this respect, understanding and controlling the crystallization process is key to fabricating organic nanocrystals with a predesigned morphology and properties [33-35]. We have reported on 2D crystalline self-assembled systems based on a hierarchical assembly mode promoted by hydrophobic and $\pi-\pi$ interactions [36]. Yet, the size and shape of these systems could not be controlled beyond the 2D morphology.

We report herein on the aqueous self-assembly of organic nanocrystals with a tunable aspect ratio. These systems are quite uniform and exhibit morphology-dependent photonics: strikingly, divergent exciton diffusion properties as a function of the shape.

\section{Results and Discussion}

Following our interest in the self-assembly of PDI derivatives, we employed compound $\mathbf{1}$, a PDI system with a hydrophilic group (phenoxybenzoic acid) attached to the aromatic core of
PDI at the "bay area" [35]. Compound $\mathbf{1}$ is an asymmetric amphiphile that was designed to result in arrays that differ from fibrous and monolayer structures assembled from symmetrically substituted PDI systems [36-38]. Additionally, the aqueous self-assembly incorporating carboxylic acid groups in the covalent unit design has been shown to result in complex and tunable self-assembly modes [39-42].

\section{Crystalline self-assembly}

We have found that the nonclassical crystallization of $\mathbf{1}$ in neutral aqueous solutions can be manipulated to result in different polymorphs [35], 3D crystals with dissimilar structures and morphologies. We envisaged that the crystallization of $\mathbf{1}$ in a basic aqueous medium can lead to $2 \mathrm{D}$ arrays (bilayers) due to the higher solubility of the assemblies as a result of the charged carboxylate groups that are expected to favor the solvation by water. We induced the self-assembly process by injecting a concentrated solution of $\mathbf{1}$ in THF $\left(2 \times 10^{-3} \mathrm{M}\right)$ into basic water ( $\mathrm{pH} 10)$ or a water/THF mixture to reach a $1 \times 10^{-4} \mathrm{M}$ concentration. We studied the following three assembly conditions: " $10 \%$ THF", i.e., injection of the stock solution to a basicwater/THF mixture to obtain a $10 \%$ THF content by volume; " $5 \%$ THF", i.e., injection of the stock solution to basic water to give a $5 \%$ THF content; " $5 \% \rightarrow 0 \%$ THF", i.e., injection of the stock solution to basic water to result in a 5\% THF content, followed by the immediate evaporation of THF in a high-vacuum and adding water to reach a $1 \times 10^{-4} \mathrm{M}$ concentration.

The self-assembly is instantaneous, as indicated by a color change from bright orange to pink in all systems. UV-vis spectra of compound 1 in aqueous medium exhibit a 0-0/0-1 vibronic band inversion, red shift, and significant broadening in comparison to the molecularly dissolved system (Figure 1B). This is a typical spectral signature of ordered PDI systems and crystals [38,43-47] having a face-to-face orientation of the $\pi$-systems.

Cryogenic transmission electron microscopy (cryo-TEM) and TEM studies revealed that the $10 \%$ THF assemblies were long rectangular-shaped crystals, $\approx 3 \mu \mathrm{m}$ in length (Figure $2 \mathrm{~A}$ ). The crystals had an aspect ratio of $2.6 \pm 1.3$, and their crystalline order was evident from FFT analysis, exhibiting well-defined spots corresponding to a periodicity of $1.6 \mathrm{~nm}$. The $5 \% \mathrm{THF}$ assembly gave rise to crystals that were $\approx 1 \mu \mathrm{m}$ in length, with an aspect ratio of $5.0 \pm 1.9$. FFT analysis revealed the spacing value to be $1.6 \mathrm{~nm}$, identical to the $10 \%$ THF system (Figure 2B). For the system $5 \% \rightarrow 0 \%$ THF, the crystals were over $5 \mu \mathrm{m}$ long and under $0.5 \mu \mathrm{m}$ in width, and thus having the largest aspect ratio amongst the studied systems, $10 \pm 3.5$. The crystalline order gave rise to a $1.6 \mathrm{~nm}$ spacing, as indicated by FFT (Figure 2C). The aspect ratio values and the calculated 

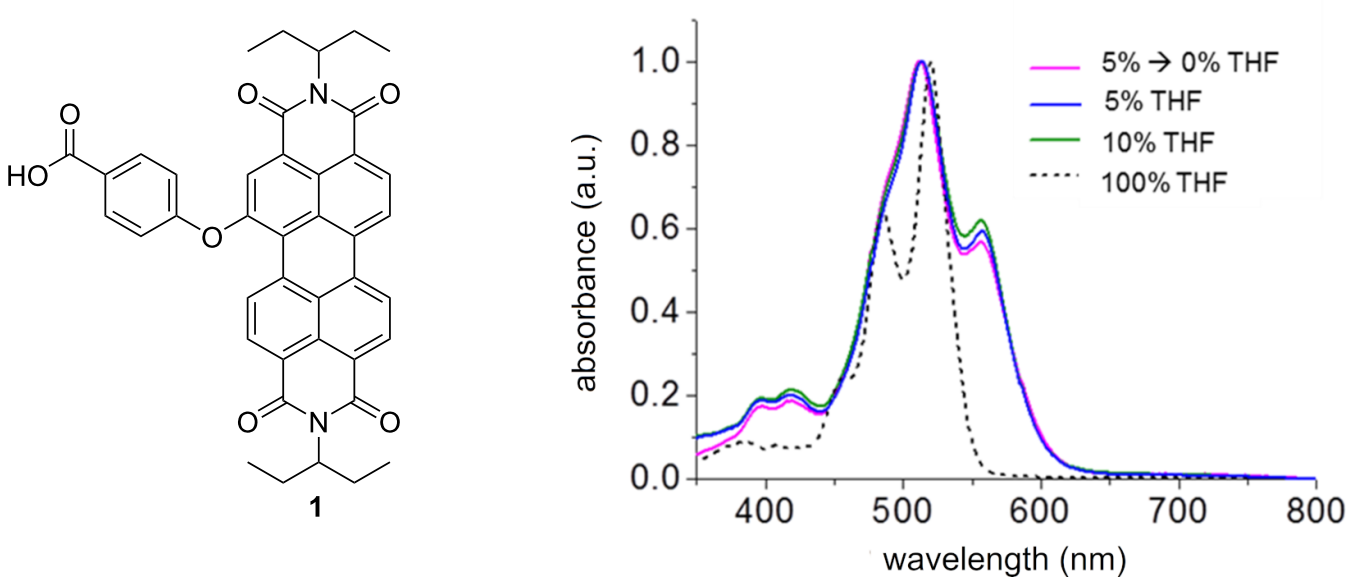

Figure 1: Chemical structure of compound 1 and UV-vis spectra in an aggregating aqueous medium and in the disaggregating solvent THF.
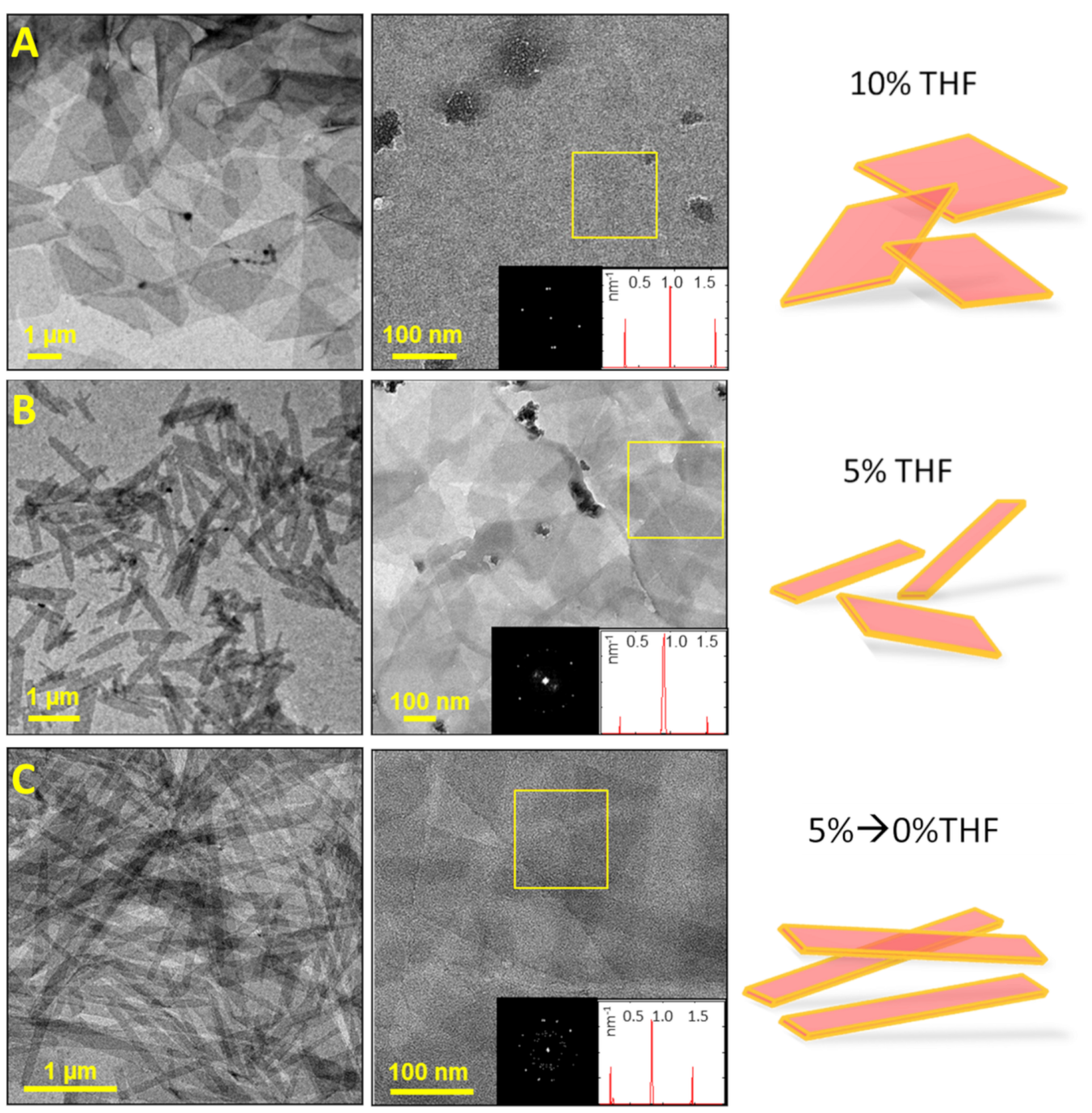

\section{$5 \% \rightarrow 0 \% \mathrm{THF}$}

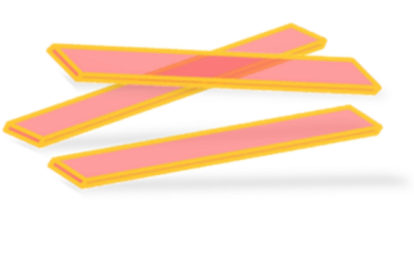

Figure 2: Transmission electron microscopy (TEM) images (left, zoomed-out and zoomed-in; $1 \times 10^{-4} \mathrm{M}$ solutions of 1 deposited on TEM grids) and crystal morphology sketches (right). A) $10 \%$ THF. B) $5 \%$ THF. C) $5 \% \rightarrow 0 \%$ THF. Insets: fast Fourier transform (FFT) analysis of the crystalline material (in the marked areas), demonstrating an identical spacing of $1.6 \mathrm{~nm}$ in all systems. 
standard deviations are based on two different assembly solutions including 50 crystals for each system.

All obtained crystalline assemblies demonstrated structural stability toward drying, and presented an identical fine structure composed of an ordered array of alternating dark and lightcontrast stripes, as observed by TEM and cryo-TEM (Figure 2 and Figure 3, respectively). FFT indicates crystallinity (welldefined spots) and identical spacings in all systems. Image analysis revealed $0.96 \pm 0.10 \mathrm{~nm}$ dark-contrast stripes separated by $0.69 \pm 0.12 \mathrm{~nm}$ light-contrast stripes in all cases. The molecular model that best fits the cryo-TEM and TEM data is a bilayer, where pairs of PDI cores are coupled together, and the carboxylic groups of both PDIs turn outwards to the surrounding aqueous medium. The resulting 1D $\pi$-stacked bilayer structures interact via the ethylpropyl residues to form an extended bilayered crystalline sheet (Figure 3C,D,E). According to the model (Figure 3C,D,E), dark-contrast stripes correspond to the overlapping aromatic cores $(1.1 \mathrm{~nm})$, and the light-contrast stripes correspond to the ethylpropyl residues $(0.6 \mathrm{~nm})$, as observed in the cryo-TEM images (Figure $3 \mathrm{~A}$ and Figure 3B).

In order to validate the bilayer width of the nanocrystals, atomic force microscopy (AFM) and SAXS measurements of the assembled compound $\mathbf{1}$ were employed. An air-dried sample of compound 1 deposited on a Si substrate (see Supporting Information File 1) gave rise to rectangular structures with welldefined edges (Figure S3, Supporting Information File 1), $3.2 \pm 0.4 \mathrm{~nm}$ in height. This is in good agreement with the bilayer width in the molecular model, corresponding to the oxygen-oxygen distance between the carboxylic groups of two adjacent PDIs (Figures S2B and S2C, Supporting Information File 1). SAXS (of a $6 \times 10^{-4} \mathrm{M}$ aqueous solution of $\mathbf{1}, 5 \% \mathrm{THF}$, Figure S4, Supporting Information File 1) demonstrated a power law dependence of $\mathrm{q}^{-2}$ in the low q region, indicative of flat particles [48]. The background subtracted curve could be fit to a dilute lamellar form factor (Equation S3, Supporting Information File 1) to give an overall thickness of $35 \AA \pm 3 \AA$, which is in excellent agreement with the molecular modeling and the AFM results.

The presented crystalline structures are kinetically trapped products since they do not equilibrate upon changing the assembly conditions after they are fully formed. Thus, the addition of THF to preassembled $5 \% \rightarrow 0 \%$ THF crystals up to a $5 \%$ THF content did not result in any observable morphological change (Figure S6B, Supporting Information File 1). Similarly, when THF was added to the assembled 5\% THF material to result in a $10 \%$ THF content, no change was observed (Figure S6A, Supporting Information File 1). Evidently, the structure of the crystals is defined by the assembly pathway rather than the equilibration at a given solvent composition. Apparently, hydrophobic interactions dominate the self-assembly, mitigating the repulsion of the charged carboxylate groups (partial protonation of the carboxylate moieties can also not be ruled out as a result of the hydrophobic self-assembly). We noted that at a lower pH (Figure S8, Supporting Information File 1), the selfassembly leads to different structures, probably due to the lower solubility and interactions between the protonated carboxylic groups. Crystals assembled under different $\mathrm{pH}$ conditions (Figure S8, Supporting Information File 1) are less homogeneous and demonstrate a larger size distribution than the crystals obtained by exerting control through the THF content.

In order to gain insight into the crystallization mechanism and the effect of the THF concentration on the crystallization process, we performed cryo-TEM imaging of the early assembly stages. For the 5\% THF assembly, monomolecular 1D stacks $1.5 \pm 0.3 \mathrm{~nm}$ in width were observed after 1 min of aging (Figure 4A). The assembled material appeared as short fibers (10-80 nm length), some of which interact and align. For the $10 \%$ THF system, crystalline arrays $\approx 100 \mathrm{~nm}$ in length and $\approx 10 \mathrm{~nm}$ in width were observed after $1 \mathrm{~min}$ (Figure $4 \mathrm{~B}$ ). The structural differences between the 5\% THF and 10\% THF systems at early assembly times reveal the distinct dynamics of the nucleation and growth process. Thus, the 10\% THF system shows a faster ordering process (THF renders the system more dynamic, lowering the activation barrier for a molecular reorganization [38,49]), resulting in long rigid fibers that interact (Figure 3E), leading to larger ordered arrays and templating the further assembly process. This, together with the better stabilization of the aromatic cores at a higher THF content, leads to large, low-aspect-ratio crystals in the $10 \%$ THF system. Thus, kinetically controlled pathway-dependent nucleation and growth defines the outcome of the crystalline self-assembly process. In both the 5\% THF and $10 \%$ THF systems, the crystallization is largely completed after 5 min (Figure S7, Supporting Information File 1). The observed crystallization pathways are consistent with a gradual order evolution mechanism recently observed by us [34].

\section{Exciton dynamics}

In order to investigate whether the morphology affects the photonic properties, we studied the excited state dynamics of the nanocrystals. Femtosecond transient absorption spectra of all assemblies displayed typical PDI excited state absorption peaks in the range of 600-770 $\mathrm{nm}$ [50], matching PDI bleaching represented by negative features at $550-600 \mathrm{~nm}$. In the disaggregated state, almost no power dependence was observed. The decay kinetics of the $5 \%$ THF and $5 \% \rightarrow 0 \%$ THF systems demonstrated power dependence (Figure 5A and Figure S9A, Supporting Information File 1, respectively). In contrast, 10\% 

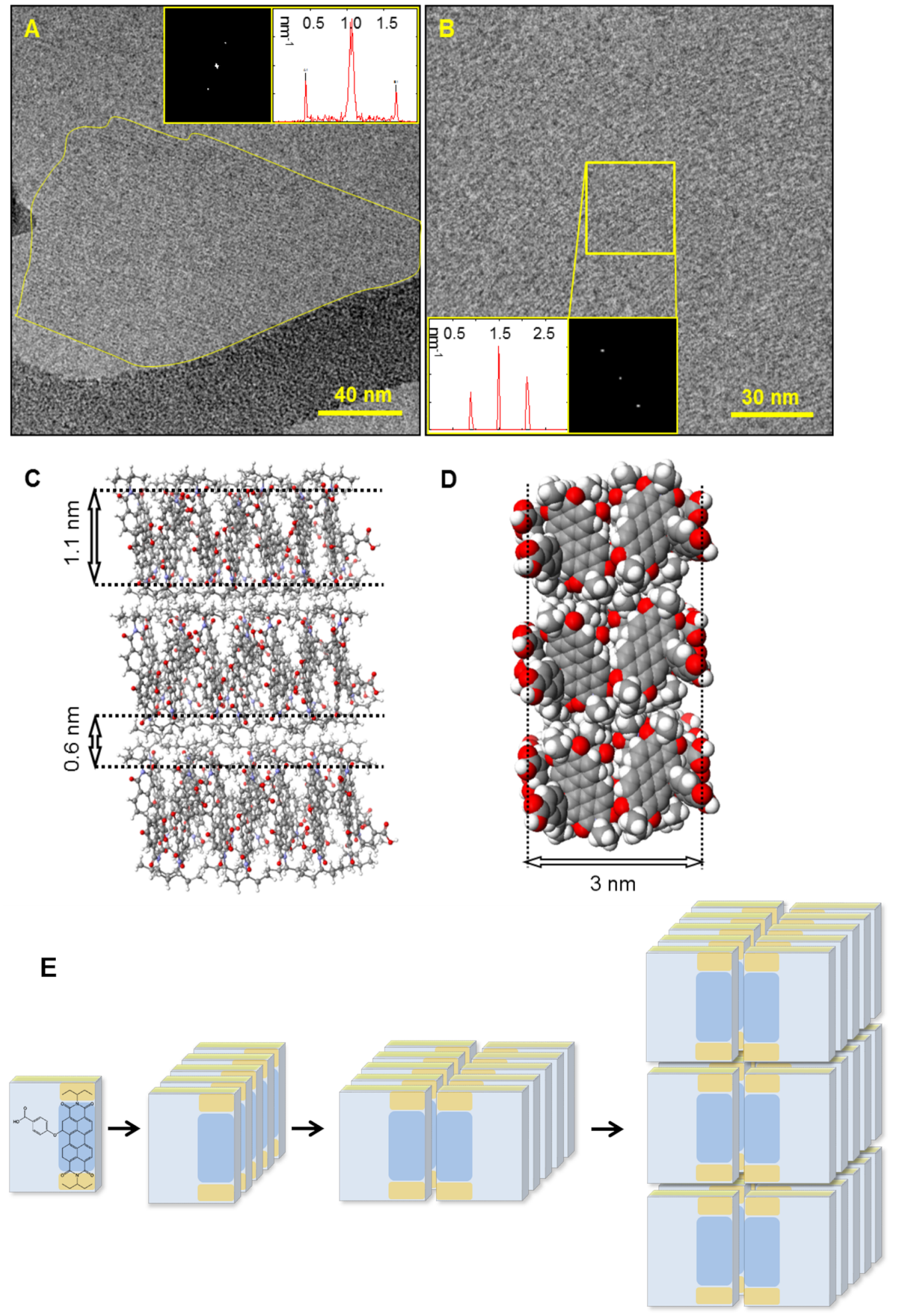

Figure 3: Cryo-TEM images of a $1 \times 10^{-4} \mathrm{M}$ solution of 1 (5\% THF) and the corresponding molecular model as well as a cartoon illustrating of the self-assembly process. A) Cryo-TEM image, the crystal is highlighted with a yellow contour. B) Zoom-in showing the fine-structure composed of alternating dark- and light-contrast stripes. Insets: FFT analysis of the crystalline material, giving rise to a spacing value of $1.6 \mathrm{~nm}$. C) and D) Molecular model: the interacting stacks and their dimensions corresponding to the observed fine-structure $(\mathrm{C})$ and a cross-section demonstrating the bilayer structure composed of two adjacent PDI cores (D). The measured distance of $3 \mathrm{~nm}$ corresponds to the oxygen-oxygen distance between the carboxylic groups of two PDIs. E) Schematic representation of the assembly structuring. 

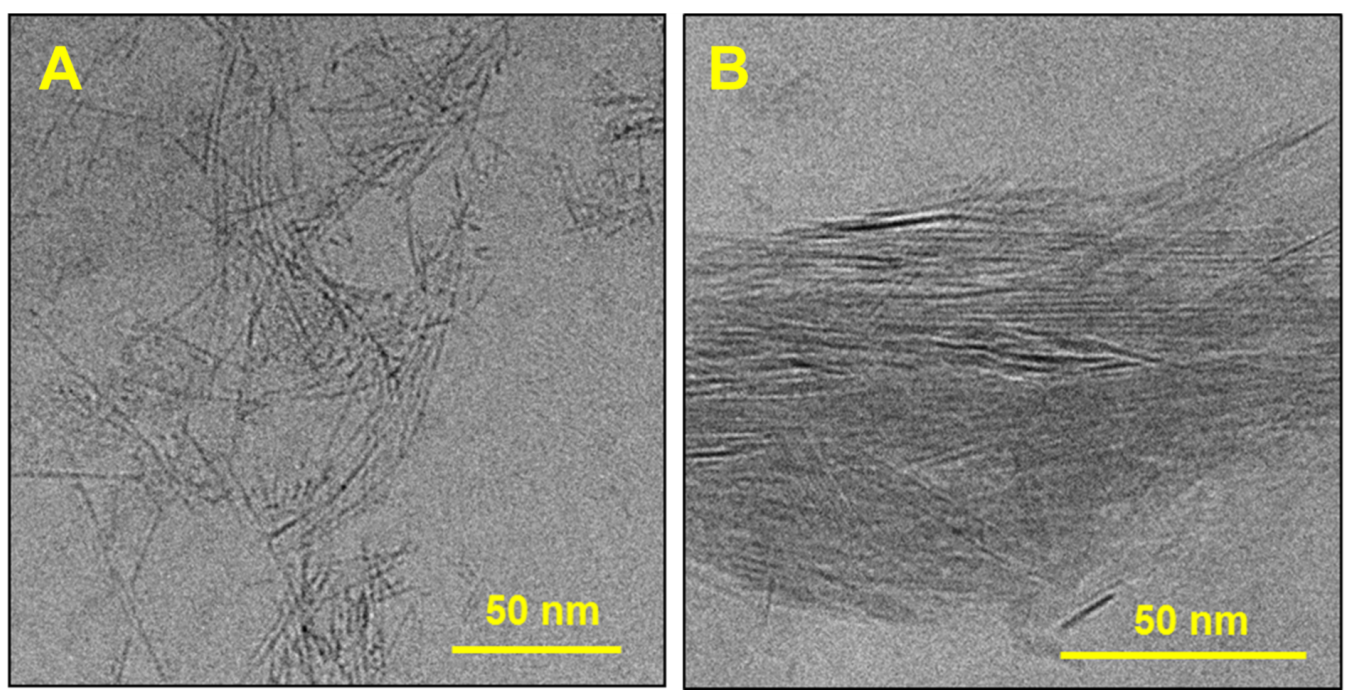

Figure 4: Cryo-TEM images of $1 \times 10^{-4} \mathrm{M}$ compound 1 in THF/water solutions after one minute of aging. A) $5 \%$ THF: monomolecular $1 \mathrm{D}$ m-stack fibers and B) $10 \%$ THF: crystalline platelets.

A

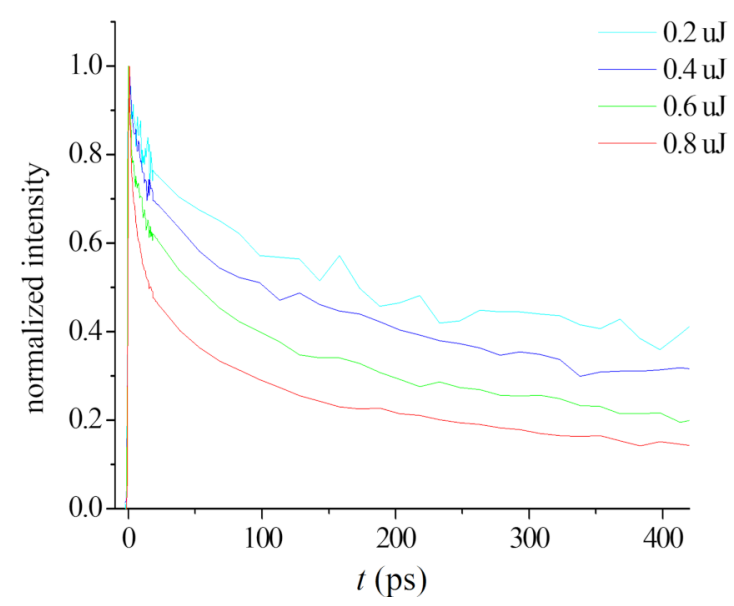

B

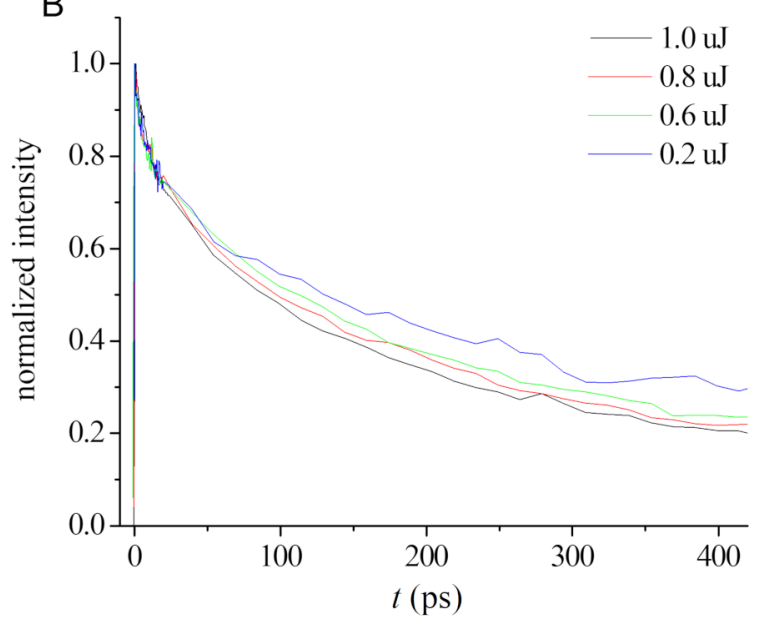

Figure 5: Transient kinetics at different laser powers probed at $755 \mathrm{~nm}\left(1 \times 10^{-4} \mathrm{M}\right.$ solution at $\left.\mathrm{pH} 10\right)$ : A) $5 \%$ THF solution and B) $10 \%$ THF solution.

THF nanocrystals exhibit essentially power-independent kinetics (Figure 5B). Power dependence is indicative of the exciton-exciton annihilation process, occurring when a high photon flux results in multiple excitons, which can efficiently diffuse through the aggregated material, resulting in exciton annihilation [51].

In order to validate the observed behavior and prove that the measured differences between the 5\% THF and 10\% THF assemblies stem from the different crystal morphologies and not from the differences in the THF concentration, a control experiment was performed. A sample of $\mathbf{1}$ prepared in 5\% THF was aged for two days, after which the THF concentration was adjusted to $10 \%$. The control sample was measured, showing exciton dynamics very similar to the original 5\% THF sample (Table 1 and Figure S9B, Supporting Information File 1).

In order to estimate the exciton diffusion coefficient and the diffusion length, we used an analysis method employed in our previous work [36] and based on a 1D diffusion model for the annihilation rate [52-55]. The model fits our data, revealing that exciton diffusion occurs primarily within 1D aromatic stacks, in agreement with the reported annihilation studies [54,55]. The fitted data is presented in Figures S10-S12, Supporting Infor- 
mation File 1, and the exciton diffusion parameters are given in Table 1.

The $5 \%$ THF and $5 \% \rightarrow 0 \%$ THF systems gave rise to similar diffusion coefficients: $5 \times 10^{-2} \mathrm{~cm}^{2} / \mathrm{s}$ and $7 \times 10^{-2} \mathrm{~cm}^{2} / \mathrm{s}$, respectively. These values are comparable to the reported exciton diffusion coefficients of 2D PDI crystalline arrays reported by us [36] and those of perylenetetracarboxylic dianhydride (PTCDA) solid crystalline films $\left(4 \times 10^{-2} \mathrm{~cm}^{2} / \mathrm{s}\right)$ [41]. The exciton diffusion lengths in the assembled material are also comparable or higher than the reported values for 2D PDI crystals $(120 \mathrm{~nm})$ [36], PTCDA films (61 nm) [52], and PDI J-aggregates $(96 \mathrm{~nm})[56,57]$, indicating efficient exciton hopping. The $10 \%$ THF assembly showed almost no power dependence, in striking difference to other systems. This can be attributed to the exciton trapping [24], probably at the large aromatic "edges" in the $10 \%$ THF crystals.

Superresolution fluorescence microscopy measurements were carried out in order to further study the photonic behavior of the systems. Interestingly, the 5\% THF system displayed an emission that was localized at regions that appeared to be matching the shape and dimensions of the crystal edges (Figure S13, Supporting Information File 1), with a clear preference for emission at the crystal corners (the microscopy of the $10 \%$ THF system did not provide reliable superresolution images). This further underscores the influence of the crystal morphology on the photonic properties [58] and will be a subject of future studies.

\section{Conclusion}

We have demonstrated that a simple building block assembles into well-defined free-floating crystals in an aqueous medium. The crystalline assemblies are stable and their morphology can be fine-tuned as a function of the initial THF concentration or the $\mathrm{pH}$ in the assembly solution. The nanocrystals demonstrate morphologically-dependent photonic properties, showing uniquely dissimilar exciton diffusion behavior. Facile fabrication of well-defined nanocrystals combined with the ability to control their morphology and photonic properties represents a convenient structure/function tool advancing the applicability of organic nanocrystals.

\section{Experimental \\ General information}

The ${ }^{1} \mathrm{H}$ and ${ }^{13} \mathrm{C}$ NMR spectra were recorded at $20{ }^{\circ} \mathrm{C}$ on a $300 \mathrm{MHz}$ NMR spectrometer (Bruker). Electrospray ionization (ESI) mass spectrometry was performed using a Micromass Platform instrument. UV-vis absorption and fluorescence measurements were carried out on a Cary-5000 spectrometer (Varian) and a Cary Eclipse fluorimeter (Varian), respectively.

\section{Preparation of the assemblies}

Samples of compound $\mathbf{1}$ were prepared by dissolving the dry material in THF, followed by the injection of the THF solution into double-distilled water (Barnstead NANOpure Diamond water system, used for all sample preparations) at the desired $\mathrm{pH}$ (adjusted by adding a $\mathrm{NaOH}$ solution). The $5 \% \rightarrow 0 \%$ THF samples were prepared by an immediate evaporation of the THF cosolvent, followed by dilution with water to the desired final concentration. Clear homogeneous pink solutions were obtained.

\section{TEM}

TEM imaging was performed using a Tecnai T12 transmission electron microscope operated at $120 \mathrm{kV}$.

Sample preparation: $8 \mu \mathrm{L}$ of each sample was applied to a 400 mesh copper grid coated with carbon (on nitrocellulose support) and blotted 2 min later from the back side of the grid.

\section{Cryo-TEM}

Cryo-TEM imaging was performed by a methodology analogous as described previously [36], using a Tecnai F20 transmission electron microscope operating at $200 \mathrm{kV}$ and using a Gatan 626 cooling holder and a transfer station with a Gatan US4000 CCD digital camera or a Tecnai T12 transmission electron microscope operated at $120 \mathrm{kV}$, using a Gatan 626 cooling holder and transfer station, with a TVIPS F244HD CCD digital camera.

Table 1: Diffusion coefficient and exciton diffusion length.

\begin{tabular}{llc} 
measured crystal system & $D, \mathrm{~cm}^{2} / \mathrm{s}$ & $L_{\mathrm{D}}, \mathrm{nm}^{\mathrm{a}}$ \\
\hline $5 \% \rightarrow 0 \%$ THF & $0.07 \pm 0.01$ & $198 \pm 4$ \\
$5 \%$ THF & $0.05 \pm 0.01$ & $170 \pm 13$ \\
pre-aged 5\% THF adjusted to 10\% THF & $0.04 \pm 0.01$ & $114 \pm 14$ \\
$10 \%$ THF & no power dependence \\
\hline
\end{tabular}

aThe exciton diffusion length is calculated using $L_{D}=D \cdot T$, where $\mathrm{T}$ is the exciton lifetime (estimated as a longer decay component of the decay fit, representing a lower limit for the exciton lifetime). 
Sample preparation: $8 \mu \mathrm{L}$ of each sample was applied to a 300 mesh copper grid coated with holey carbon (Pacific GridTech supplies). The samples were blotted at $25{ }^{\circ} \mathrm{C}$ and $95 \%$ relative humidity and plunged into liquid ethane using a Leica EM-GP Automatic Grid Plunger. The specimens were equilibrated at $-178{ }^{\circ} \mathrm{C}$ in the microscope prior to the imaging process. Time-dependent cryo-TEM samples were prepared just before the plunging, using a stopwatch. The same assembly solution that was plunged at $t=1$ min was used again at $t=5 \mathrm{~min}$ and $t=30 \mathrm{~min}$ in order to elucidate the structural evolution of the self-assembled crystals.

\section{Femtosecond transient absorption}

Femtosecond transient absorption spectroscopy was performed by a methodology analogous as described previously [36], using a system based on a mode-locked Ti:sapphire oscillator (Spectra Physics MaiTai). The oscillator produces a train of $<120 \mathrm{fs}$ pulses (bandwidth $\approx 10 \mathrm{~nm}$ FWHM), with a peak wavelength centered at $800 \mathrm{~nm}$. The weak oscillator pulses are amplified by a chirped pulse regenerative amplifier (Spectra Physics Spitfire ACE). The pulses are first stretched, then regeneratively amplified in a Ti:sapphire cavity, pumped by a pulsed Nd:YLF laser (Spectra Physics Empower 45) operating at $1 \mathrm{kHz}$. After the pulse has been amplified and recompressed, the energy is about $5.0 \mathrm{~mJ}$ in a train of $1 \mathrm{kHz}$ pulses and about $1 \mathrm{~mJ}$ is used in the transient absorption setup. An independent pump pulse is obtained by pumping an optical parametric amplifier (Spectra Physics OPA-800CF) that produces $120 \mathrm{fs}$ pulses tunable from $300 \mathrm{~nm}$ to $3 \mu \mathrm{m}$. The output power of the OPA is a few microjoule (depending on the chosen wavelength) at $1 \mathrm{kHz}$. The pump beam is mechanically chopped at half the amplifier repetition rate. The chopper (C-995 TTI) is synchronized with the Spitfire pulses. Normally, a few thousand pulse pairs (pump on/ pump off) are averaged to produce a transient absorption spectrum with a noise level below $0.3 \mathrm{mOD}$. A small portion of the remaining amplified pulse is used to generate a white-light continuum as a probe pulse. To this end, the Ti:sapphire beam is focused onto a $3 \mathrm{~mm}$ thick sapphire disk by a $10 \mathrm{~cm}$ focal length lens, and the numerical aperture of the beam is controlled by an iris placed in front of the lens to obtain a stable and smooth white-light continuum. The resulting beam is passed through a Raman notch filter in order to remove the remains of the $800 \mathrm{~nm}$ fundamental beam from the probe whitelight continuum. The pump and probe pulses are crossed in the sample at a small angle while maintaining a magic angle between the pump and probe polarizations. The remains of the pump pulse are removed by passing the probe through an iris, and it is then imaged onto an optical fiber that brings it into a fiber optic interface, which focuses the light onto the entrance slit of a Jobin Yivon Triax 180 spectrograph. The light is normally dispersed by a $300 \mathrm{gr} / \mathrm{mm}$ grating onto a fast CCD camera (Andor Newton DU-970N-UV, operating at 1,000 spectra per second using "crop mode"). The whole setup is controlled by the National Instruments LabView software. A variable neutral-density filter was employed to adjust the pump power for studying the power dependence. The pump power intensities were measured using an Ophir powermeter with a photodiode sensor in proximity to the sample. The excitation densities were calculated for a laser spot of $300 \mu \mathrm{m}$ diameter on the sample. This diameter was measured by placing a beamprofiler (Ophir Beamstar FX33) at the sample position and determination the 4 -sigma ( $95 \%$ of the power) parameter. In the reported experiments, the pump was tuned to 525 or to $590 \mathrm{~nm}$, and the optical densities of the samples (in $4 \mathrm{~mm}$ and $2 \mathrm{~mm}$ optical path length cuvettes) were kept between 0.2 and 0.5 at the excitation wavelength. The instrument response function (300 fs) was recorded by repetition of the experiments, with the sample replaced by the pure solvent and keeping all other parameters unchanged. Spectral corrections and analysis were performed using the Surface Xplorer Pro (Ultrafast Systems) and Origin 7.5 (OriginLab) softwares.

\section{Supporting Information}

\section{Supporting Information File 1}

General information, synthesis, molecular modeling, and further experimental details.

[https://www.beilstein-journals.org/bjoc/content/ supplementary/1860-5397-17-5-S1.pdf]

\section{Funding}

This work was supported by grants from the Israel Science Foundation, Minerva Foundation, Schmidt Minerva Center for Supramolecular Architectures, and the Helen and Martin Kimmel Center for Molecular Design.

\section{ORCID ${ }^{\circledR}$ iDs}

Gilad Haran - https://orcid.org/0000-0003-1837-9779

Boris Rybtchinski - https://orcid.org/0000-0002-2071-8429

\section{Preprint}

A non-peer-reviewed version of this article has been previously published as a preprint: https://doi.org/10.3762/bxiv.2020.100.v1

\section{References}

1. Brus, L. Appl. Phys. A: Solids Surf. 1991, 53, 465-474. doi:10.1007/bf00331535

2. Kelly, K. L.; Coronado, E.; Zhao, L. L.; Schatz, G. C. J. Phys. Chem. B 2003, 107, 668-677. doi:10.1021/jp026731y 
3. Alivisatos, A. P. Science 1996, 271, 933-937. doi:10.1126/science.271.5251.933

4. Halperin, W. P. Rev. Mod. Phys. 1986, 58, 533-606. doi:10.1103/revmodphys.58.533

5. Peng, X.; Schlamp, M. C.; Kadavanich, A. V.; Alivisatos, A. P. J. Am. Chem. Soc. 1997, 119, 7019-7029. doi:10.1021/ja970754m

6. Colvin, V. L.; Schlamp, M. C.; Alivisatos, A. P. Nature 1994, 370 , 354-357. doi:10.1038/370354a0

7. Kasai, H.; Nalwa, H. S.; Oikawa, H.; Okada, S.; Matsuda, H.; Minami, N.; Kakuta, A.; Ono, K.; Mukoh, A.; Nakanishi, H. Jpn. J. Appl. Phys., Part 1 1992, 31, L1132-L1134. doi:10.1143/jjap.31.I1132

8. lida, R.; Kamatani, H.; Kasai, H.; Okada, S.; Oikawa, H.; Matsuda, H. Kakuta, A.; Nakanishi, H. Mol. Cryst. Liq. Cryst. Sci. Technol., Sect. A 1995, 267, 95-100. doi:10.1080/10587259508033979

9. Fu, H.-B.; Wang, Y.-Q.; Yao, J.-N. Chem. Phys. Lett. 2000, 322, 327-332. doi:10.1016/s0009-2614(00)00419-x

10. Kasai, H.; Kamatani, H.; Okada, S.; Oikawa, H.; Matsuda, H.; Nakanishi, H. Jpn. J. Appl. Phys., Part 1 1996, 35, L221-L223. doi:10.1143/jjap.35.I221

11. Fu, H.-B.; Yao, J.-N. J. Am. Chem. Soc. 2001, 123, 1434-1439. doi:10.1021/ja0026298

12. Baba, K.; Kasai, H.; Masuhara, A.; Oikawa, H.; Nakanishi, H. Jpn. J. Appl. Phys. 2009, 48, 117002. doi:10.1143/jjap.48.117002

13. Fery-Forgues, S. Nanoscale 2013, 5, 8428-8442. doi:10.1039/c3nr02657d

14. Nakanishi, H.; Katagi, H. Supramol. Sci. 1998, 5, 289-295. doi:10.1016/s0968-5677(98)00021-2

15. Rosenne, S.; Grinvald, E.; Shirman, E.; Neeman, L.; Dutta, S.; Bar-Elli, O.; Ben-Zvi, R.; Oksenberg, E.; Milko, P.; Kalchenko, V.; Weissman, H.; Oron, D.; Rybtchinski, B. Nano Lett. 2015, 15, 7232-7237. doi:10.1021/acs.nanolett.5b02010

16. Schierl, C.; Niazov-Elkan, A.; Shimon, L. J. W.; Feldman, Y.; Rybtchinski, B.; Guldi, D. M. Nanoscale 2018, 10, 20147-20154. doi:10.1039/c8nr04155e

17. Kasai, H.; Murakami, T.; Ikuta, Y.; Koseki, Y.; Baba, K.; Oikawa, H.; Nakanishi, H.; Okada, M.; Shoji, M.; Ueda, M.; Imahori, H.; Hashida, M. Angew. Chem., Int. Ed. 2012, 51, 10315-10318. doi:10.1002/anie.201204596

18. Zhao, Y. S.; Fu, H.; Peng, A.; Ma, Y.; Xiao, D.; Yao, J. Adv. Mater. (Weinheim, Ger.) 2008, 20, 2859-2876. doi:10.1002/adma.200800604

19. Komai, Y.; Kasai, H.; Hirakoso, H.; Hakuta, Y.; Okada, S.; Oikawa, H.; Adschiri, T.; Inomata, H.; Arai, K.; Nakanishi, H. Mol. Cryst. Liq. Cryst. Sci. Technol., Sect. A 1998, 322, 167-172. doi:10.1080/10587259808030217

20. Jiang, H.; Zhang, K. K.; Ye, J.; Wei, F.; Hu, P.; Guo, J.; Liang, C.; Chen, X.; Zhao, Y.; McNeil, L. E.; Hu, W.; Kloc, C. Small 2013, 9, 990-995. doi:10.1002/smll.201202390

21. Hiremath, R.; Basile, J. A.; Varney, S. W.; Swift, J. A. J. Am. Chem. Soc. 2005, 127, 18321-18327. doi:10.1021/ja0565119

22. Kang, J. F.; Zaccaro, J.; Ulman, A.; Myerson, A. Langmuir 2000, 16, 3791-3796. doi:10.1021/la9914054

23. Briseno, A. L.; Aizenberg, J.; Han, Y.-J.; Penkala, R. A.; Moon, H.; Lovinger, A. J.; Kloc, C.; Bao, Z. J. Am. Chem. Soc. 2005, 127 12164-12165. doi:10.1021/ja052919u

24. Kim, B. J.; Yu, H.; Oh, J. H.; Kang, M. S.; Cho, J. H. J. Phys. Chem. C 2013, 117, 10743-10749. doi:10.1021/jp400807t

25. Balakrishnan, K.; Datar, A.; Oitker, R.; Chen, H.; Zuo, J.; Zang, L. J. Am. Chem. Soc. 2005, 127, 10496-10497. doi:10.1021/ja052940v
26. Balakrishnan, K.; Datar, A.; Naddo, T.; Huang, J.; Oitker, R.; Yen, M.; Zhao, J.; Zang, L. J. Am. Chem. Soc. 2006, 128, 7390-7398. doi:10.1021/ja061810z

27. Zhang, Z.; Zhang, X.; Zhan, C.; Lu, Z.; Ding, X.; He, S.; Yao, J. Soft Matter 2013, 9, 3089-3097. doi:10.1039/c2sm27674g

28. Che, Y.; Datar, A.; Balakrishnan, K.; Zang, L. J. Am. Chem. Soc. 2007, 129, 7234-7235. doi:10.1021/ja071903w

29. Weissbuch, I.; Lahav, M.; Leiserowitz, L. Cryst. Growth Des. 2003, 3 , 125-150. doi:10.1021/cg0200560

30. Vekilov, P. G. Cryst. Growth Des. 2010, 10, 5007-5019. doi:10.1021/cg1011633

31. Jehannin, M.; Rao, A.; Cölfen, H. J. Am. Chem. Soc. 2019, 141, 10120-10136. doi:10.1021/jacs.9b01883

32. Sear, R. P. Int. Mater. Rev. 2012, 57, 328-356. doi:10.1179/1743280411y.0000000015

33. Davey, R. J.; Schroeder, S. L. M.; ter Horst, J. H. Angew. Chem., Int. Ed. 2013, 52, 2166-2179. doi:10.1002/anie.201204824

34. Tsarfati, Y.; Rosenne, S.; Weissman, H.; Shimon, L. J. W.; Gur, D.; Palmer, B. A.; Rybtchinski, B. ACS Cent. Sci. 2018, 4, 1031-1036. doi:10.1021/acscentsci.8b00289

35. Shahar, C.; Dutta, S.; Weissman, H.; Shimon, L. J. W.; Ott, H.; Rybtchinski, B. Angew. Chem., Int. Ed. 2016, 55, 179-182. doi:10.1002/anie.201507659

36. Shahar, C.; Baram, J.; Tidhar, Y.; Weissman, H.; Cohen, S. R.; Pinkas, I.; Rybtchinski, B. ACS Nano 2013, 7, 3547-3556. doi:10.1021/nn400484y

37. Krieg, E.; Rybtchinski, B. Chem. - Eur. J. 2011, 17, 9016-9026. doi:10.1002/chem.201100809

38. Krieg, E.; Niazov-Elkan, A.; Cohen, E.; Tsarfati, Y.; Rybtchinski, B. Acc. Chem. Res. 2019, 52, 2634-2646. doi:10.1021/acs.accounts.9b00188

39. Matern, J.; Dorca, Y.; Sánchez, L.; Fernández, G. Angew. Chem., Int. Ed. 2019, 58, 16730-16740. doi:10.1002/anie.201905724

40. Matsumoto, N. M.; Lafleur, R. P. M.; Lou, X.; Shih, K.-C.; Wijnands, S. P. W.; Guibert, C.; van Rosendaal, J. W. A. M.; Voets, I. K.; Palmans, A. R. A.; Lin, Y.; Meijer, E. W. J. Am. Chem. Soc. 2018, 140, 13308-13316. doi:10.1021/jacs.8b07697

41. Basak, D.; Ghosh, S. ACS Macro Lett. 2013, 2, 799-804. doi: $10.1021 / \mathrm{mz} 400357 \mathrm{~g}$

42. Yin, M.; Shen, J.; Pisula, W.; Liang, M.; Zhi, L.; Müllen, K. J. Am. Chem. Soc. 2009, 131, 14618-14619. doi:10.1021/ja9058662

43. Cormier, R. A.; Gregg, B. A. Chem. Mater. 1998, 10, 1309-1319. doi:10.1021/cm970695b

44. Kazmaier, P. M.; Hoffmann, R. J. Am. Chem. Soc. 1994, 116, 9684-9691. doi:10.1021/ja00100a038

45. Klebe, G.; Graser, F.; Hädicke, E.; Berndt, J. Acta Crystallogr., Sect. B: Struct. Sci. 1989, 45, 69-77. doi:10.1107/s0108768188010407

46. Zang, L.; Che, Y.; Moore, J. S. Acc. Chem. Res. 2008, 41, 1596-1608. doi:10.1021/ar800030w

47. Würthner, F.; Bauer, C.; Stepanenko, V.; Yagai, S. Adv. Mater. (Weinheim, Ger.) 2008, 20, 1695-1698. doi:10.1002/adma.200702935

48. Glatter, O.; Kratky, O. Small angle $x$-ray scattering; Academic Press: London, U.K., 1982. doi:10.1002/actp.1985.010360520

49. Tidhar, Y.; Weissman, H.; Wolf, S. G.; Gulino, A.; Rybtchinski, B. Chem. - Eur. J. 2011, 17, 6068-6075. doi:10.1002/chem.201003419 
50. Wasielewski, M. R. J. Org. Chem. 2006, 71, 5051-5066. doi:10.1021/jo060225d

51. Pope, C. E.; Swenberg, M. Electronic Processes in Organic Crystals and Polymers; Oxford University Press: Oxford, U.K., 1999.

52. Engel, E.; Leo, K.; Hoffmann, M. Chem. Phys. 2006, 325, 170-177. doi:10.1016/j.chemphys.2005.09.004

53. Ahrens, M. J.; Sinks, L. E.; Rybtchinski, B.; Liu, W.; Jones, B. A.; Giaimo, J. M.; Gusev, A. V.; Goshe, A. J.; Tiede, D. M.; Wasielewski, M. R. J. Am. Chem. Soc. 2004, 126, 8284-8294. doi:10.1021/ja039820c

54. Suna, A. Phys. Rev. B 1970, 1, 1716-1739.

doi:10.1103/physrevb.1.1716

55. Inoue, A.; Yoshihara, K.; Nagakura, S. Bull. Chem. Soc. Jpn. 1972, 45 1973-1976. doi:10.1246/bcsj.45.1973

56. Marciniak, H.; Li, X.-Q.; Würthner, F.; Lochbrunner, S. J. Phys. Chem. A 2011, 115, 648-654. doi:10.1021/jp107407p

57. Rehhagen, C.; Stolte, M.; Herbst, S.; Hecht, M.; Lochbrunner, S.; Würthner, F.; Fennel, F. J. Phys. Chem. Lett. 2020, 11, 6612-6617. doi:10.1021/acs.jpclett.0c01669

58. Bisri, S. Z.; Takenobu, T.; Yomogida, Y.; Shimotani, H.; Yamao, T.; Hotta, S.; Iwasa, Y. Adv. Funct. Mater. 2009, 19, 1728-1735. doi:10.1002/adfm.200900028

\section{License and Terms}

This is an Open Access article under the terms of the Creative Commons Attribution License (https://creativecommons.org/licenses/by/4.0). Please note that the reuse, redistribution and reproduction in particular requires that the author(s) and source are credited and that individual graphics may be subject to special legal provisions.

The license is subject to the Beilstein Journal of Organic Chemistry terms and conditions:

(https://www.beilstein-journals.org/bjoc/terms)

The definitive version of this article is the electronic one which can be found at:

https://doi.org/10.3762/bjoc. 17.5 